\title{
Reliability of Kinovea Computer Program in Measuring Cervical Range of Motion in Sagittal Plane
}

\author{
Shereen H. Elwardany ${ }^{1,2 *}$, Wadida H. El-Sayed ${ }^{3}$, Mohammad F. Ali2,4 \\ ${ }^{1}$ Physical Therapy Department, Kasr El Aini Eleiny, Cairo University Hospitals, Cairo, Egypt \\ ${ }^{2}$ Physical Therapy Department, College of Medical Rehabilitation, Qassim University, Buridah, Saudi Arabia \\ ${ }^{3}$ Basic Science Department, Faculty of Physical Therapy, Cairo University, Cairo, Egypt \\ ${ }^{4}$ Orthopedic Physical Therapy Department, Faculty of Physical Therapy, October 6 University, Giza, Egypt \\ Email: shereenwerdany@gmail.com, dr.wadidaHassan@gmail.com, Farouq.farouk@gmail.com
}

Received 3 September 2015; accepted 20 September 2015; published 28 September 2015

Copyright (C) 2015 by authors and OALib.

This work is licensed under the Creative Commons Attribution International License (CC BY).

http://creativecommons.org/licenses/by/4.0/

(c) (i) Open Access

\begin{abstract}
Purpose: This study was conducted to investigate the intrarater and interrater reliability of Kinovea computer program in measuring cervical range of motion in sagittal plane in healthy subjects. Subjects: Sixty-five normal physical therapy intern students participated in this study. Their age ranged from $20-24$ years with mean $(22.42 \pm 0.84)$ and BMI mean $(28.60 \pm 1.40)$. Materials and Methods: Digital camera and labtop with installed Kinovea software were used. The cervical ROM of flexion and extension for each participant were measured by Kinovea computer program three times by examiner (B) to detect the intrarater reliability and by three examiners (A, B and C) to detect the interrater reliability. Results: For the intrarater reliability, the ICC between the first and second trials by Rater B for flexion and extension were 0.985 and 0.995 . The ICC between the second and third trials were 0.932 and 0.993 . The ICC between the first and third trials were 0.920 and 0.986 respectively. For the interrater reliability, the ICC between Rater A \& B for flexion and extension were 0.991 and 0.992 . The ICC between Rater $A \& C$ were 0.988 and 0.9938 . The ICC between Rater B \& C were 0.993 and 0.997 respectively. Kinovea computer program had an excellent intrarater and interrater reliability in measuring cervical flexion and extension range of motion at $P<0.05$. Conclusion: The Kinovea software program was reliable in both intrarater and interrater reliability in measuring cervical range of motion in sagittal plane.
\end{abstract}

\section{Keywords}

Reliability, Kinovea, Cervical Range of Motion, Cervical Disorders

Subject Areas: Orthopedics

\footnotetext{
${ }^{*}$ Corresponding author.
}

How to cite this paper: Elwardany, S.H., El-Sayed, W.H. and Ali, M.F. (2015) Reliability of Kinovea Computer Program in Measuring Cervical Range of Motion in Sagittal Plane. Open Access Library Journal, 2: e1916. 


\section{Introduction}

Cervical disorders are major health problems and an important source of disability. The incidence of cervical problems around the world has a considerable impact on societal health. The clinical manifestations of cervical disorders include pain, headache and decreased range of motion (ROM) which may reduce social participation [1].

Assessment of ROM is a key concern for the physical therapist when evaluating a patient presenting with cervical disorders. Valid and reliable assessment tools are necessary [2].

Reliability of ROM measurement tool means the consistency or the repeatability of the same ROM measurements, and expresses their reproducibility or stability only in relation to the time intervals reported [3]. The most accurate evaluation of the reliability of the instrument and procedures is determined when short time intervals separate tests, the classic “test-retest" study design [3] [4].

Kinovea computer program is a software that is able to measure ROM of the joints of the body; the overview function is a summary image of the video. It samples images from the video at regular interval and creates a composite picture where you can see the motion at a glance. The reverse function lets you play the motion back ward [5]. Goiometers and inclinometers were used to measure cervical ROM. The CROM is one of the most used and recent tools among clinicians which has a good validity and reliability. However, it is a relatively expensive instrument [6] [7].

The most affected cervical ROM in mechanical neck dysfunction are extension and rotation [8] [9]. There is need to have a new, accurate, easily applicable and low cost method for measuring the cervical ROM. There is need to have an assessment method that can be utilized in the evaluation of therapeutic modalities effect [4] and also provide a method of documentation. This will help the physical therapist to make informed judgments about patient's physical impairments as well as patient's response to treatment in cervical disorders.

The aim of this study was to investigate the intrarater and interrater reliability of Kinovea computer program in measuring cervical ROM in sagittal plane.

\section{Methods}

This study was conducted in the Faculty of Physical Therapy, Cairo University 2015. Repeated measurement design was used in this study to investigate the intrarater and interrater reliability of Kinovea computer program in measuring active cervical range of motion (ACROM) in sagittal plane. Sixty five healthy normal physical therapy intern students participated in this study, their age ranged from 20-24 years and BMI less than 30 from both genders; they were selected by convenient sampling. Each subject was given full explanation, verbal instructions concerning the purpose and procedure of the current study and the participants signed a consent form with local ethics guidelines prior to participating. All volunteers did not receive monetary rewards or compensation for their time and participation to this study. All volunteers were assessed by the same instruments and the same observers. Ethical approval was obtained from the faculty.

\subsection{Inclusion Criteria}

- Sixty five normal subjects (physical therapy intern students) with asymptomatic neck pain and ROM deficits.

- Age of participants 20 - 24 years.

- Both genders.

- BMI less than 30 .

\subsection{Exclusion Criteria}

- Previous pain or muscle spasm 3 month before measurements.

- Previous history of musculoskeletal or neurological problems of the neck region.

- Previous history of cervical trauma, bone pathologies, arthritis, cervical rib, forward head posture or other inflammatory disorders.

- Any patient with the diagnosis of spasmodic torticollis was excluded.

\subsection{Instrumentations}

\section{1) Digital Camera}




\section{CANON A-810 16M 5X HD BK}

TFT screen LCD, Screen size 2.7 inch, Max resolution $4608 \times 3456$ Px. Effective pixels 16 MB Sensor type Super HAD CCD Sensor size 1/2.3-inch Image ratio w:h (4:3), (16:9).

\section{2) Tripod Stand}

The digital camera was placed on adjustable tripod stand for standardization. Its height 1.5 meter and the distance between the tripod and the feet of participant was 1.5 meter.

\section{3) Kinovea Software Program}

Kinovea is video analysis software dedicated to sport. It targets primarily medical professionals, the coaches and athletes. The supported files are displayed. You can save the videos easily and access it later in need. The video can be analyzed in slow motion so the video can be seen by frame by frame. Lines and arrows can be added on the video with the drawing tool [5]. Kinovea computer program was installed on the computer before applying the measurement procedure.

\section{4) Computer System}

HP PAVILION G6 LAPTOP

Processor CPU Speed 2.5, cache memory 3MB, RAM memory FSB 1333 MHZ, RAM up to 8 GB, hard drive capacity 750, rotational speed 5400RPM, graphic card, HD Bright view LED-backlit with $1366 \times 768$ screen resolution, size 15.6 inch.

Laptop was used for the Kinovea measurement analysis.

5) Markers

Round markers of $1.5 \mathrm{~cm}$ in diameter were glued on special bony land marks.

\section{6) Chest Belt}

It was used for fixation and standardization.

\subsection{Selection of Examiners for the Reliability Study}

\section{The Interrater Reliability}

To detect the interrater reliability; three physical therapist examiners (A, B and C) assessed the cervical ROM measurements. They had graduated from the same university; Faculty of Physical Therapy, Cairo University. (Rater A physical therapist specialist with 15 year experience, Rater B physical therapist specialist with 20year experience and Rater $\mathrm{C}$ physical therapist with 10 years experience. They had no previous experience with the Kinovea computer program before applying the experiment. Before the beginning of the study, the examiners received three hours of training to adequately manipulate the Kinovea computer program under supervision of highly experienced physical therapist with the Kinovea. In their training sessions, they were taught how to determine which anatomical point of reference which the markers placed on with the Kinovea computer program and they trained for an hour to make sure their method was standardized. Then they measured cervical ROM with the Kinovea computer program as a three trials before starting the Kinovea computer program measurement analysis. All raters were blinded to the results of each other's reading.

\section{The Intrarater Reliability}

To detect the intrarater reliability rater B was used for the intrarater reliability. This rater assessed all subjects on three separate occasions with a 48 hour period separated. The intraclass correlation coefficient (ICC) was calculated between each two trials.

\section{Evaluation Procedure}

- Physical examination for the neck region was done before the experiment to exclude any disease or neurological deficits that may affect the cervical ROM measurements. History

- Inspection from front, lateral and posterior aspects of the neck region.

- Palpation for the neck region and related areas.

- Special tests (compression-distraction-Adeson) test were applied in doubt cases to confirm and give accurate decision about the participant inclusion.

- Group muscle test for neck muscles.

\section{A) Camera Setup}

The position of the camera was fixed for standardization all over the experiment and was determined on the basis of the position of the subjects. The position of the tripod on the floor. The position of the subject is marked also and the distance between the participant feet and the tripod was measured by the tape measurement to avoid any changes affect the measurement. 


\section{B) Application of Markers}

The participant wore 2 elastic headbands to affix head markers. Markers were placed on centered over the external acoustic meatus and the vertex [11].

\section{Measurement Procedure}

Each subject was sitting on a stool close to a wall while maintaining good upright balanced posture, with both feet on the floor, with normal lumbar lordosis, hands on thighs, and with 90 degrees in the hip and knee joints. Each subject was requested to assume a neutral head position, with the purpose of positioning the head's center of mass in a vertical plane through the atlantooccipital joints with the nose pointing forward in line with the sternum and bellybutton [12] [13].

Each subject was given full explanation, verbal instruction concerning the purpose and procedure of the study. Markers were placed on the vertex and centered over the external acoustic meatus [11].

The digital camera was steady to record before position the subject.

\section{Measuring Cervical ROM by Kinovea Computer Program}

For the purpose of this study, participants were simply asked to perform maximal (end-range) neck forward flexion with hold 5 seconds then neutral position and lastly extension with hold 5seconds.

\section{Cervical Flexion and Extension}

Cervical flexion and extension ROM were measured on a sagittal plane. A marker centered over the external acoustic meatusis the axis of angle between two line segments [11]. The first line segment between the axis and the marker placed on the vertex. The second line is the vertical line on marker placed over the external acoustic meatus after patient did chin in. The position for each marker was indicated with a washable ink pen for accurate replacement then the marker was glued on the ink pen [14].

- The examiner was sitting behind the camera taking the video.

- The examiner asked the participant to flex his neckthen hold 5 seconds at the end of the rangeand return to the neutral position as shown in Figure 1.

- From neutral position the examiner asked the participant to extend his neck then hold 5 seconds at the end of the range and return to the neutral position as shown in Figure 2.

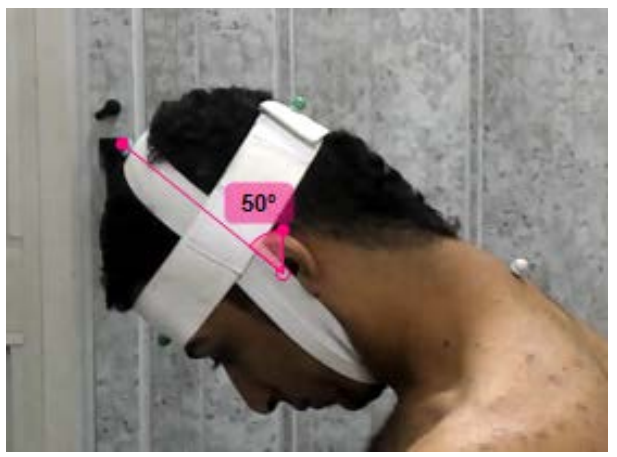

Figure 1. Cervical flexion by Kinovea.

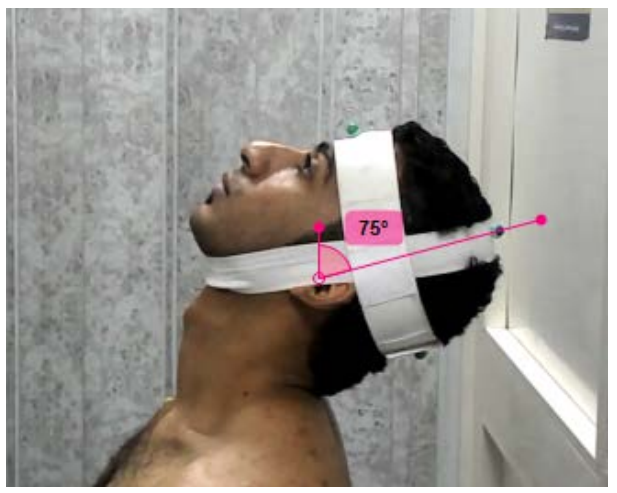

Figure 2. Cervical extension by Kinovea. 
- The examiner asked the participant to do cervical flexion with chin tuck and extension with chin elevation.

- The procedure was repeated three times.

The examiner recorded the results after doing the analysis of each video of each participant by Kinovea computer program.

C) Analysis the Range of Motion by the Kinovea Computer Program as Follow:

- The examiner connected the camera with computer.

- The examiner apply the analysis for each cervical ROM measurements in sagittal plane.

- The procedure was repeated three times then the mean average of the measured angles by Kinovea program were recorded.

- The analysis of each participant were saved on computer in a separate file.

\subsection{Statistical Analysis}

1) Descriptive statistics (mean and standard deviation) for measurements of the cervical flexion and extension ROM by Kinovea computer program.

2) Intra-class correlation coefficient (ICC) was used to estimate the intrarater reliability.

3) Intra-class correlation coefficient (ICC) was used to detect the interrater reliability of Kinovea program. The confidence Interval was $(\mathrm{CI}=95 \%)$ with $(P<0.05)$ for all tests. For the reliability part of the study, intra and interrater reliability were estimated with the intraclass correlation coefficient (ICC). ICC is a statistic designed to measure the size and direction of the association between two variables [15]. The data were analyzed by SPSS version 20 .

\section{Results}

In this study, 65 subjects (54 male and 11 female) were assigned into one group and as shown in Table 1, their mean age was $(22.42 \pm 0.84)$ years, their mean weight was $(73.78 \pm 6.43) \mathrm{kg}$, their mean height was $(160.23 \pm$ $6.17) \mathrm{cm}$ and their mean BMI was $(28.60 \pm 1.40)$. ICC $>0.4$ was regarded as moderate and ICC $>0.7$ as high correlation [15].

\section{Intra-rater reliability}

To calculate the inter-rater reliability, the mean average of flexion and extension ROM value for each movement were compared between each two trials. Table 2 shows the mean and SD of flexion and extension and the ICC in the first and second trial in Rater B (Flexion ICC 0.985-Extension ICC0.995). Table 3 shows the mean and SD of flexion and extension and the ICC in the second and third trials in Rater B (Flexion ICC 0.932-Extension ICC 0.993). Table 4 shows the mean and SD of flexion and extension and the ICC in the first and third trials in Rater B (Flexion ICC 0.920-Extension ICC00.986). Cervical flexion and extension movements had an excellent reliability.

\section{Inter-rater reliability}

To calculate the inter-rater reliability, the mean average of flexion and extension ROM value for each movement were compared between each two Raters. Mean and SD of flexion, extension and the ICC between Rater A \& B (Flexion ICC 0.991-Extension ICC 0.992) as shown in Table 5. Mean and SD of flexion, extension and the ICC between Rater A \& C (Flexion ICC. 988-Extension ICC 0.9938) as shown in Table 6. Mean and SD of flexion, extension and the ICC between Rater B \& C. (Flexion ICC0.993-Extension ICC 0.997) as shown in Table 7). Kinovea software program had an excellent inter-rater reliability in measuring cervical range of motion in sagittal plane.

\section{Table 1. Physical characteristics of the subjects.}

\begin{tabular}{ccc}
\hline Variables & Mean & \pm SD \\
\hline Age (yrs) & 22.42 & \pm 0.84 \\
Weight (Kg) & 73.78 & \pm 6.43 \\
Height (cm) & 160.23 & \pm 6.17 \\
BMI $\left(\mathbf{K g} / \mathbf{m}^{\mathbf{2}}\right)$ & 28.60 & \pm 1.40 \\
\hline
\end{tabular}


Table 2. Intraclass correlation between first and second trials for (Rater B).

\begin{tabular}{cccc}
\hline Cervical movement & $\mathbf{1}^{\text {st }}$ trial $($ Mean \pm SD) & $\mathbf{2}^{\text {nd }}$ trial $($ Mean \pm SD) & ICC \\
\hline Flexion & $58.83 \pm 9.327$ & $59.15 \pm 9.154$ & 0.985 \\
Extension & $72.91 \pm 6.887$ & $72.68 \pm 6.856$ & 0.995 \\
\hline
\end{tabular}

Table 3. Intraclass correlation between second and third trials for (Rater B).

\begin{tabular}{cccc|}
\hline Cervical movement & $\mathbf{2}^{\text {nd }}$ trial $($ Mean \pm SD) & 3d trial $($ Mean \pm SD) & ICC \\
\hline Flexion & $58.83 \pm 9.327$ & $60.14 \pm 8.972$ & 0.932 \\
Extension & $72.68 \pm 6.856$ & $72.58 \pm 6.899$ & 0.993 \\
\hline
\end{tabular}

Table 4. Intraclass correlation between first and third trials for (Rater B).

\begin{tabular}{cccc}
\hline Cervical movement & $\mathbf{1}^{\text {st }}$ trial $($ Mean \pm SD) & 3d trial $($ Mean \pm SD) & ICC \\
\hline Flexion & $58.83 \pm 9.327$ & $60.14 \pm 8.972$ & 0.920 \\
Extension & $72.91 \pm 6.887$ & $72.58 \pm 6.899$ & 0.986 \\
\hline
\end{tabular}

Table 5. Mean and SD of flexion, extension and ICC between (Rater A \& B).

\begin{tabular}{cccc}
\hline Cervical movement & Rater A (Mean \pm SD) & Rater B (Mean \pm SD) & ICC C.I. 95.00\% \\
\hline Flexion & $58.70 \pm 9.37$ & $59.37 \pm 8.98$ & $0.991(0.985-0.994)$ \\
Extension & $72.81 \pm 6.88$ & $72.72 \pm 6.86$ & $0.996(0.994-0.998)$ \\
\hline
\end{tabular}

Table 6. Mean and SD of flexion, extension and ICC between (Rater A \& C).

\begin{tabular}{ccccc}
\hline Cervical movement & Rater A (Mean \pm SD) & Rater C (Mean \pm SD) & ICC C.I. 95.00\% & $0.9889(0.981-0.993)$ \\
\hline Flexion & $58.70 \pm 9.37$ & $59.6923 \pm 8.95636$ & $72.8154 \pm 6.88724$ & $0.9938(0.989-0.996)$ \\
\hline Extension & $72.81 \pm 6.88$ & 724 & \\
\hline
\end{tabular}

Table 7. Mean and SD of flexion, extension and ICC between (Rater B \& C).

\begin{tabular}{cccc}
\hline Cervical movement & Rater B (Mean \pm SD) & Rater C (Mean \pm SD) & ICC C.I. 95.00\% \\
\hline Flexion & $59.37 \pm 8.98$ & $59.6923 \pm 8.95$ & $0.993(0.989-0.996)$ \\
Extension & $72.72 \pm 6.86$ & $72.8154 \pm 6.88$ & $0.997(0.995-0.998)$ \\
\hline
\end{tabular}

\section{Discussion}

This study is the first to examine the Intra-rater and Inter-rater reliability of Kinovea software program in measuring cervical ROM in sagittal plane. Clinical studies have shown that impaired neck rotation, extension, and neck retraction predict high disability [8]-[10]. Professionals dealing with the seissues have been seeking a reliable instrument for measuring all cervical motions [12].

For a measurement instrument to be useful, the two most important factors that must be established are reliability and validity which affecting the objective measurements [4].

Kinovea software program has many advantages. It is an accurate method that can be utilized in the establishment of normal values of cervical flexion and extension ROM. It can be utilized in the evaluationprocess in patients with cervical disorders. It is low cost modality. It needs minimal training.

Kinovea software has some disadvantages. It is 2D not 3D program. The therapists who will use it needed to be trained before measuring. There is need to have camera and laptop with installed Kinovea.

Because limited studies are available on the Kinovea, the validity and reliability of this software program 
were necessary to be evaluated.

\section{Intra-rater reliability}

Reliability estimates very important psychometric properties since before an instrument can be considered valid, it needs to be reliable [6]. Examiner B was chosen for the intrarater reliability because he is the most experienced one. Three trials were done by examiner B to estimate the ICC. The findings of this study showed that when the cervical flexion and extension ROM were measured with the Kinovea computer program by the same examiner (examiner B), similar results were found from one session to the next. In the sagittal plane, an excellent reliability was found for flexion and extension ROM between the first and second trial (Flexion ICC 0.985-Extension ICC 0.995; 95\% CI), the second and third (Flexion ICC 0.932-Extension ICC 0.993; 95\% CI), and the first and third trial in Rater B (Flexion ICC 0.920-Extension ICC 0.986; 95\% CI).

\section{Inter-rater reliability}

When the cervical flexion and extension ROM were measured by three independent examiners the results were compared, the ICCs were excellent for movements in the sagittal plane and great agreements between the Raters were found. The assessment of the examiner's skills showed that they were competent (ICC >0.65) in the use of the method [9]. The ICC between Rater A \& B (Flexion ICC 0.991-Extension ICC 0.992; 95\% CI). The ICC between Rater A \& C (Flexion ICC 0.988-Extension ICC 0.993; 95\% CI). The ICC between Rater B \& C (Flexion ICC00.993-Extension ICC 0.997; 95\% CI).

Tousignant et al. [16] who investigate the intratester and intertester reliability of the cybex electronic digital inclinometer (EDI-320) for measurement of active neck flexion and extension in healthy subjects, reported flexion (ICC $=0.77$; 95\%CI: $0.62-0.87$ ) and extension (ICC $=0.83$; 95\% CI: $0.63-0.92$ ) using the EDI-320. However, this device is not on the market anymore. In comparison, the reliability coefficients were found slightly higher in the current study.

Laflame et al., [6] who investigate the reliability and validity of i phone in measuring cervical ROM. The results of that study revealed moderate inter-rater reliability for movements in the sagittal (ICCs $=0.48-0.49$ ). The differences in results between the study done by Tousignant et al. [16] and laflame et al., [6] might be explained by the fact that the first took the measurements with the inclinometer while the subjects were insupine position, whereas the other study used the compass rather than the inclinometer of the iPhone. Since the compass is not influenced by gravity, but rather by orientation of the iPhones, it has more potential source of error than the inclinometer, which could have easily influenced theintra and inter-rater ICCs. Furthermore, the magnetometer which serves as the hardware for the compass application is more sensible of the presence of electromagnetic fields which is another factor that could contribute to the lower ICCs for the measurements of neck rotation in that study. In comparison, in the current study higher ICCs were found in both intrarater and interrater reliability when measuring cervical flexion and extension ROM because the cervical flexion and extension ROM by Kinovea computer program were not affected by gravity or these other factors affecting the results of those studies.

Youdas et al., [11] determine reliabilities within and between persons measuring cervical active range of motion (AROM), three methods were examined: use of a cervical-range of motion (CROM) instrument, use of a universal goniometer (UG), and visual estimation (VE). For the intrarater reliability, the high ICC values indicate that AROM measurements of neck flexion $(\mathrm{KC}=0.95)$ and extension (ICC $=0.90)$ obtained with the CROM device are highly reproducible when repeated by the same physical therapist. The UG measurements demonstrated flexion (ICC $=0.86$ ) and extension (ICC =0.83).The inter-tester reliability for AROM measurements of neck flexion and extension (ICCs $=0.86$ ) for the CROM device, whereas the UG demonstrated flexion $(\mathrm{ICC}=0.57)$ and $(\mathrm{ICC}=0.79)$ for extension.

Tucci et al., [17] reported between-tester reliability for neck flexion (ICC $=0.08$ ), although they reported reliability for neck extension (ICC $=0.82$ ) when healthy subjects were measured by two experienced examiners using a UG. With VE, the between-tester reliability for AROM measurements was poor (ICC $=0.42$ ) for both flexion and extension.

The universal goniometer is the most cheap and available in the markets and clinics. However, the therapist faced many problems during the measurement procedure with the UG, firstly keeping the reference arm of the goniometer stationary while rotating the joint seem to be difficult. It also may be difficult to read the goniometer at end ROM. Removing the goniometer from the joint to read the value can result in unintended movement of the goniometer. [4] in addition its lower ICC [7].

The systematic review of Williams et al., [7] showed that the CROM is one of the most used and recent tools 
among clinicians which has a good validity when compared for all cervical spine movements (ICCs $=0.82$ 0.98 ) and reliability (ICCs $=0.58-0.99$ ) when compared to a gold standard (X-ray). However, it is a relatively expensive.

In the current study higher ICCs were estimated in cervical flexion and extension in intra and interrater reliability than any inclinometers, goniometers and any device depending on sensors or magnetic field or gravity affected. The cervical ROM was measured as angles between determined two lines by Kinovea software program. The high ICCs may be attributed good standardization of markers placement and the training sessions of the raters that allowing them to have less errors. The physical therapists need to have accurate, less expensive, easy to use tool without utilizing sensors that may need to be calibrated, to measure cervical ROM.

Other factors may contribute to the results of the current study are the motivation of the subjects, their ability to understand the instructions, and the raters' behavior, that and instruct the subjects, or the subject's ability to make and repeat the movement in the same way [12].

Guzmán et al., [2] who used Kinovea computer as a computational tools instead of traditional sensors and special hardware program in therapeutic motion analysis for the lower limbs rehabilitation. The position, velocity and acceleration in a rehabilitation for lower limbs was obtained through a new proposed method (Kinovea). The evaluation of the software and the test performed focused in the rehabilitation of lower limbs. The main purpose of that study was simply to facilitate the application of techniques for the physiotherapist. The advantages demonstrated in this article can be a starting point to analyze fast systems of multiple degrees of freedom for rehabilitation purposes. The motion detection using Kinovea evaluate the rehabilitation progress of the patient to achieve the best recovery with low cost advantage.

The results of the current study in agreement of the study done by Abdelreheem et al., [4] whoinvestigate the intra-rater and inter-rater reliabilities of the Kinovea software program for the measurement of dominant wrist joint range of motion in healthy participants. One hundred normal participants participated in this study, aged from 20 - 35 years old. Selected from both sexes (55 male and 45 female). They were recruited from the student and the employees of Al-Haram hospital, physical therapy department. subjects were selected according to the following criteria: 1) aged from 20 - 35 years old; 2) all subjects were normal; 3) grade 3 wrist muscles test. Any subject with history of musculoskeletal or neurological problems of the dominant upper extremity, and previous upper limb surgery were excluded.

The results of that study revealed that the Kinovea software program was reliable in both inter- and intra-rater reliability for Wrist joint ROM measurement.

Guzmán et al., [2] used Kinovea computer program for measurement of movement of lower limbs use one camera similarly, in the current study one camera was used and Abdelreheem et al., [4] used also one camera to determine wrist ROM by Kinovea. In contrary a study done by Lisa et al., [18] used three camera to ensure usable images for measurement and analysis in all three planes of movement.

\section{Strengths and Limitations}

First, the selected examiners were physiotherapist. Second, the three examiner's initial preparation (training) with the Kinovea computer program represents strength. Third, the selected participants in the current study were physical therapy intern students so they understand the instructions and the procedure well and performing the procedure in an excellent manner.

Fourth, standardization of the procedures also helped minimize random errors. To achieve this, all participants were stabilized in order to avoid compensation. Also, the given instructions were the same instructions before each measurement for all participants and the environment was identical during all the data collection process: same rooms, same orientation or the participants (facing east), same chairs. All subjects were tested in a standardized seated position using marker placements and non goniometric estimation techniques.

Most reliability studies used only two raters for intertester reliability [11] [12] [17]. In this study three raters were used to confirm the results. Also Abdelreheem et al., [4] had three raters.

The assessment of validity and reliability of a new ROM measurement tools is so important, as the existing tools have many disadvantages beginning from the size, price, availability ending with the reliability and the ability of physiotherapist to deal with it, so Kinovea software program now become reliable measurement tool that can be used in the physical therapy field [4].

The reliability might be improved by employing a better standardized procedure and by ensuring that the 
same instructions were given to the study subjects. The reliability for this measurement method should also be confirmed with a greater number of subjects.

\section{Limitations}

The current study was limited by small sample size.

\section{Conclusion}

The Kinovea software program was reliable in both interrater and intrarater reliability in measuring cervical range of motion in sagittal plane.

\section{Recommendations}

Further studies with large sample size. Reliability of Kinovea computer program in measuring cervical ROM in transverse and frontal planes. Validity of Kinovea computer program in measuring cervical range of motion in all directions were recommended.

\section{Acknowledgements}

The authors are thankful to all physical therapy intern volunteers from Faculty of Physical Therapy for their critical contribution in the present study.

\section{References}

[1] Buskila, D., Sarzi-Puttini, P. and Ablin, J.N. (2007) The Genetics of Fibromyalgia Syndrome. Pharmacogenomics, 8, 67-74. http://dx.doi.org/10.2217/14622416.8.1.67

[2] Guzmán, C., Valdivia, A., Blanco, O., Oliver-Salazar, M.A. and Carrera-Escobedo, J.L. (2013) Therapeutic Motion Analysis of Lower Limbs Using Kinovea. International Journal of Soft Computing and Engineering (IJSCE), 3.

[3] Gajdosik, R.L. and Bohannon, R.W. (1987) Clinical Measurement of Range of Motion: Review of Goniometry Emphasizing Reliability and Validity. Physical Therapy, 67, 1867-1872.

[4] Abd EL-Reheem, R.M., Kamel, R.M. and Ali, M.F. (2015) Reliability of Using Kinovea Program in Measuring Dominant Wrist Joint Range of Motion. Trends in Applied Sciences Research, 10, 224-230.

[5] Abass, S. and Abdulhassan, Z. (2013) Kinematic Analysis of Human Climbing up and Down Stairs at Different Inclinations. Eng.\&Tech. Journal, 31.

[6] Laflamme, Y.T., Boutin, N., Dion, N.A. and Vallée, C.A. (2013) Reliability and Criterion Validity of Two Applications of the iPhone to Measure Cervical Range of Motion in Healthy Participants. Journal of Neuroengineering and Rehabilitation, 10, 69. http://dx.doi.org/10.1186/1743-0003-10-69

[7] Williams, M.A., McCarthy, C.J., Chorti, A., Cooke, M.W. and Gates, S. (2010) A Systematic Review of Reliability and Validity Studies of Methods for Measuring Active and Passive Cervical Range of Motion. Journal of Manipulative and Physiological Therapeutics, 33, 138-155. http://dx.doi.org/10.1016/j.jmpt.2009.12.009

[8] Dawood, R.S., Kattabei, O.M., Nasef, S.A., Battarjee, K.A. and Abdelraouf, O.R. (2013) Effectiveness of Kinesio Taping versus Cervical Traction on Mechanical Neck Dysfunction. International Journal of Therapies and Rehabilitation Research, 2.

[9] Mahgoub, M.S., Abd El-Aziz, H.G., Saleh, A.M. and Hegazy, M.O. (2013) Efficacy of Kinesio Taping versus Phonophoresis on Mechanical Neck Dysfunction. International Journal of Recent Advances in Multidisciplinary Research, 01, 0128-0134.

[10] Olson, S.L., O’Connor, D. P., Birmingham, G., Broman, P. and Herrera, L. (2000) Tender Point Sensitivity, Range of Motion, and Perceived Disability in Subjects with Neck Pain. Journal of Orthopaedic and Sports Physical Therapy, 30, 13-20. http://dx.doi.org/10.2519/jospt.2000.30.1.13

[11] Youdas, J.W., Carey, J.R. and Garrett, T.R. (1991) Reliability of Measurements of Cervical Sspine Range of Motion-Comparison of Three Methods. Physical Therapy, 71, 98-104.

[12] Severinsson, Y., Elisson, L. and Bunketorp, O. (2012) Reliability of Measuring the Cervical Sagittal Translation Mobility with a Simple Method in a Clinical Setting. Rehabilitation Research and Practice, 2012, Article ID: 629104.

[13] Tousignant, M., de Bellefeuille, L., O’Donoughue, S. and Grahovac, S. (2000) Criterion Validity of the Cervical Range of Motion (CROM) Goniometer for Cervical Flexion and Extension. Spine, 25, 324-330. 
http://dx.doi.org/10.1097/00007632-200002010-00011

[14] Burns, D.K. and Wells, M.R. (2006) Gross Range of Motion in the Cervical Spine: The Effects of Osteopathic Muscle Energy Technique in Asymptomatic Subjects. The Journal of the American Osteopathic Association, 106, 137-142.

[15] Fleiss, J.L. (1986) Design and Analysis of Clinical Experiments. Willey, New York.

[16] Tousignant, M., Boucher, N., Bourbonnais, J., Gravelle, T., Quesnel, M. and Brosseau, L. (2001) Intratester and Intertester Reliability of the Cybex Electronic Digital Inclinometer (EDI-320) for Measurement of Active Neck Flexion and Extension in Healthy Subjects. Manual Therapy, 6, 235-241. http://dx.doi.org/10.1054/math.2001.0419

[17] Tucci, S.M., Hicks, J.E., Gross, E.G., et al. (1986) Cervical Motion Assessment: A New, Simple and Accurate Method. Archives of Physical Medicine and Rehabilitation, 67, 225-230.

[18] Trew, L.M. and Meyer, E.R. "Effect of a Dynamic Seating Surface on Postural Control and Function in Children with Cerebral Palsy”-Experiences Gained and Lessons Learned; Using SPCM, Pressure Mapping and Videography, M.Sc., PT 6, 441-447. 